\title{
Avaliação da eficácia de um programa de estimulação cortical para melhora da atenção de crianças com TDAH
}

Alisson Padilha de Lima ${ }^{1}$ Fabrício Bruno Cardoso² Iris Lima e Silva ${ }^{3}$ Lucianne Fragel Madeira $^{4}$ Alfred Sholl Franco ${ }^{5}$

\begin{abstract}
RESUMO
Este estudo teve como objetivo avaliar a eficácia de um programa de estimulação cortical para crianças com Transtorno de Atenção e Hiperatividade (TDAH), que expressam corporalmente a dificuldade em manter um estado de atenção concentrado durante suas aulas. Participaram do estudo 80 crianças do sexo masculino com idade compreendida entre 8 e 11 anos, diagnosticados com TDAH e que foram avaliadas pelo aparelho ERP-P300 para identificar a eficácia da intervenção após 140 sessões de estimulação cortical. Pode-se concluir que a estimulação cortical, por via auditiva, realizada por meio de batidas binaural, mostrou-se valiosa na melhora dos níveis de atenção de crianças com TDAH.
\end{abstract}

Descritores: Avaliação, Crianças, Estimulação Cortical e Transtornos de Atenção.

\section{Evaluation the effectiveness of a program of cortical stimulation to improve the attention of children witch} $A D H D$

\begin{abstract}
This study had witch objective evaluation the efficacy a program of cortical stimulation for children witch attention hyperactivity disorder (ADHD), expressing bodily difficulty in maintaining a state of focused attention during their lessons. The study included 80 male children aged between 8 and 11 years, diagnosed witch ADHD and have evaluated by the device p-300 ERP to identify the effectiveness of the intervention after 140 sessions cortical stimulation. It can be concluded that the cortical stimulation, for auditory pathway performed by binaural beats, has proved valuable in improving the level of attention in children witch ADHD.
\end{abstract}

Descriptors: Evaluation, Children, Cortical Stimulation, Attention of Disorder.

\footnotetext{
${ }^{1}$ Especialista em Educação especial pela Universidade Católica de Bom Bosco (UCDB), Campo Grande, MS, Brasil.

${ }^{2}$ Doutorando em Ciências biológicas na Universidade Federal do Rio de Janeiro (UFRJ), Rio de Janeiro, RJ, Brasil.

${ }^{3}$ Doutoranda em Neurociências na Universidade Federal Fluminense (UFF), Niterói, RJ, Brasil.

${ }^{4}$ Pós doutorado em Biofísica celular pela Universidade Federal do Rio de Janeiro (UFRJ), Rio de Janeiro, RJ, Brasil.

${ }^{5}$ Doutor em Ciências biológicas pela Universidade Federal do Rio de Janeiro (UFRJ), Rio de Janeiro, RJ, Brasil.
} 


\section{Introdução}

A atenção é um processo complexo que carece de divisão em múltiplas operações, ela é parte integrante e fundamental da atividade sensorial e é indispensável à linguagem, à aprendizagem ${ }^{1}$. E ainda participa como um distribuidor da atividade sensorial pelos vários níveis de consciência que em simultâneo processam a informação ${ }^{2}$, assim pode-se dizer que atenção é um complexo domínio neurocomportamental que se constitui como base fundamental de todas as funções cognitivas superiores ${ }^{3}$.

A sua enorme importância no amplo espectro de funções mentais reflete-se, na verdade, na grande quantidade de áreas cerebrais que a ela estão devotadas, a atenção pode então ser considerada como um "sistema orgânico", com a sua própria anatomia funcional, a sua estrutura celular e os seus circuitos específicos ${ }^{4}$.

O Transtorno de Déficit de Atenção e Hiperatividade (TDAH) é caracterizado como um padrão persistente de desatenção e/ou hiperatividade, mais freqüente e severo do que aquele observado em indivíduos em níveis significativos de desenvolvimento, assim alguns sintomas devem estar presentes antes dos 7 anos, mas muitos indivíduos são diagnosticados após a presença dos sintomas por alguns anos, os prejuízos são identificados em razão dos sintomas apresentados durante o processo de formação ${ }^{5}$.

Nos processos de memória-aprendizado, em crianças com TDAH, principalmente na aquisição de uma linguagem, as dificuldades mais comuns são na fala, na leitura e na escrita devido à presença de disfunção em uma área frontal do cérebro conhecida como região orbital frontal, a qual se constitui em uma das regiões cerebrais responsável pela inibição do comportamento, pelo controle da atenção, pelo planejamento futuro, pelos juízos e pelo autocontrole ${ }^{6}$.

Sendo assim, as crianças com TDAH apresentam problemas nas funções centrais da memória de trabalho, dificultando a realização das tarefas cognitivas, uma vez que estas requerem que os indivíduos retenham e processe a informação, isto implica em uma eficiente maquinaria neuronal responsável pelo processo de síntese de informações, indispensável ao reconhecimento do estímulo?.

É consistente considerar que a criança com TDAH apresenta alguns aspectos que podem dificultar ou mesmo limitar a aquisição e a permanência da aprendizagem em função do déficit de transferência de atenção da memória operacional para a memória de longa duração refletindo diretamente, entre outras disfunções, tais como a aquisição da linguagem lectoescrita ${ }^{8}$.

Assim o termo estimulação cortical se caracteriza através de uma técnica de estimulação visual e/ou, auditiva, voltada para a busca da predominância das ondas corticais tipo Alfa, responsáveis pela aprendizagem, onde na metodologia protocolar foi baseada nas freqüências de alfa entre 8 e $13 \mathrm{HZ}$, como normalmente utilizada em estudos nesta linha de investigação ${ }^{9}$.

Dessa forma a estimulação cortical pode ser usada para estimular ritmos elétricos no cérebro, e esses estímulos das ondas cerebrais pode ser alcançado pelas batidas binaurais ou isochronic tones, e a gama das ondas cerebrais vai de 0 a 40hz, e grande parte dessas freqüências está fora da capacidade auditiva humana, porém, existem métodos específicos para realizar este estímulo, utilizando aparelhos que geram binaural beats proporcionando modificações ou ganhos no repertório perceptivo motor do ser humano ${ }^{10}$.

A partir do comentado anteriormente este estudo teve como objetivo avaliar a eficácia de um programa de estimulação cortical para crianças com TDAH, que expressam corporalmente a dificuldade em manter um estado de atenção concentrado durante suas aulas.

\section{Metodologia}

\section{Tipologia do estudo}

O presente estudo foi desenvolvido por meio de um formato ou desenho quase experimental, considerando-se que uma pesquisa consiste em determinar um objeto de estudo, selecionar as variáveis que seriam capazes de influenciá-la, definir as formas de controle e de observação dos efeitos que a variável independente produz diretamente na variável dependente, isto sendo realizado em uma avaliação antes (pré-teste), e uma avaliação após (pós-teste) à aplicação de uma intervenção ${ }^{11}$. 


\section{Amostra}

No período de maio 2012 foram selecionadas 80 crianças do sexo masculino com idade compreendida entre 8 e 11 anos, diagnosticados com Transtorno de Atenção e Hiperatividade (TDAH), onde somente crianças do gênero masculino foram identificadas com (TDAH). Todos foram provenientes do ambulatório de uma clinica particular de neuropediatria da cidade do Rio de Janeiro. Cabe ressaltar que o diagnóstico de TDAH foi feito a partir de exames clínicos, exames laboratoriais como eletro encefalograma, além de exames de imagens como ressonância magnética e tomografia computadorizada e não faziam uso de medicação.

\section{Ética na Pesquisa}

O protocolo de pesquisa foi avaliado e aprovado pelo Comitê de Ética da Universidade Castelo Branco, sob número de registro 0172/2008. Todos os responsáveis das crianças participantes deste estudo assinaram o Termo de Consentimento Livre e Esclarecido (TECLE), concordando com a participação da criança na pesquisa.

\section{Procedimentos de Avaliação}

Para a consecução do objetivo deste estudo foram realizadas duas avaliações pelo ERP-P300, uma antes da intervenção e uma após as 140 sessões de intervenção.

O ERP-P300 foi realizado sempre pelo mesmo examinador, num mesmo ambiente, com iluminação e temperatura padronizadas, sempre no mesmo horário (9 horas da manhã), para evitar variação circadiana. Eletrodos ativos foram colocados nas posições $\mathrm{FZ}, \mathrm{CZ}$ e PZ, de acordo com o sistema internacional ${ }^{10-20}$. Eletrodos de referência foram colocados em A1 e A2, ligados entre si. $O$ eletrodo terra foi posicionado em FPZ. Foram também colocados eletrodos acima da sobrancelha direita e no canto externo do olho esquerdo para controle do eletro-oculograma. 0 paradigma utilizado foi o "oddball". Os estímulos auditivos foram apresentados bilateralmente através de fones de ouvido com tons de $70 \mathrm{~dB}$ e dois tons foram apresentados de forma aleatória: estímulo raro, de $1500 \mathrm{~Hz}$ (com 20\% de probabilidade de aparecimento) e estímulo freqüente, de $800 \mathrm{~Hz}$ (com $80 \%$ de probabilidade de aparecimento). O intervalo entre os estímulos foi fixado em 2 segundos. Foi pedido ao testado que apertasse um botão todas as vezes que escutasse um som raro, com a mão dominante, tão rapidamente quanto possível. Foram obtidas médias de 30 registros de estímulos raros, livres de artefatos. Todos os procedimentos foram repetidos, para permitir a replicação das ondas obtidas e facilitar a identificação dos componentes.

\section{Procedimentos de Intervenção}

Realizado por meio da estimulação cerebral, em nível cortical, o procedimento de intervenção iniciou-se dois dias após a avaliação e foi realizada durante 140 sessões de 40 minutos cada uma, onde o objetivo foi de realizar um treinamento mental a partir de um estado alterado de consciência, produzido pelas batidas binaural, por isso, programou-se para as crianças participantes deste estudo que fossem estimuladas com uma freqüência entre 8 e $10 \mathrm{HZ}^{9}$. Onde cada sessão teve a seguinte anatomia:

$$
\begin{aligned}
& \text { 0min/2min - 8hz } \\
& 2 \mathrm{~min} / 3 \mathrm{~min}-10 \mathrm{hz} / 8 \mathrm{hz} \\
& 3 \mathrm{~min} / 38 \mathrm{~min}-8 \mathrm{hz} / 9 \mathrm{hz} \\
& 38 \mathrm{~min} / 40 \mathrm{~min}-8 \mathrm{hz} / 10 \mathrm{hz}
\end{aligned}
$$

A referida intervenção ocorreu em uma sala ampla e arejada da própria clinica, onde as crianças recebiam um aparelho de CD Player da marca Brithania, com fones de ouvido estéreos, além de um CD com toda programação adequada, sendo orientada a permanecer deitado em uma cama, com fones estéreos, no ouvido, conectados ao aparelho de Cd player.

Cabe ressaltar que a intervenção ocorreu de forma individual e sempre na mesma sala e no mesmo horário, das 9 horas da manhã.

\section{Tratamento estatístico}

A análise dos dados deste estudo foi realizada com base na comparação de resultados estatísticos utilizando-se 0 programa de estatística BIOESTAT 5.0. Os resultados obtidos pelas avaliações foram calculados através da estatística descritiva, a média e o desvio padrão. Todos os dados foram considerados paramétricos, pelo teste de T-STUDENT, onde p valor $<0,05$. 


\section{Resultados}

Na primeira avaliação o desempenho das crianças participantes deste estudo teve sua variação entre 432 e 602 ms, com uma média de $490.11 \mathrm{~ms}$, já na segunda avaliação a variação de resultados ficou entre 403 e 562 ms, com uma média igual a $459.88 \mathrm{~ms}$, o que tendência assim a uma melhora na velocidade de respostas destas crianças após o período de intervenção da estimulação cerebral.

Tabela I: Resultados obtidos do grupo nos dois instantes pré e pós intervenção.

\begin{tabular}{l|l|l}
\hline ESPECIFICIDADES & PRÉ AVALIAÇÃO & PÓS AVALIAÇÃO \\
\hline Mínimo & 432.00 & 403.00 \\
\hline Máximo & 602.00 & 562.00 \\
\hline Mediana & 482.00 & 451.50 \\
\hline Primeiro Quartil (25\%) & 460.75 & 434.75 \\
\hline Média Aritmética & 490.11 & 459.88 \\
\hline Desvio Padrão & 41.25 & 37.71 \\
\hline Coeficiente de Variação & $8.42 \%$ & $8.20 \%$ \\
\hline
\end{tabular}

Fonte: Dados da Pesquisa

Ao se analisarem este resultado através do teste estatístico teste T de Student foi obtido que a diferença de $30.23 \mathrm{~ms}$ entre as duas médias das avaliações desenvolvidas neste estudo é significante estatisticamente pois foi revelado para um $t=24.25$ um $p=0.0001$, ou sejas as crianças melhoraram sua capacidade de atenção concentrada em função da intervenção e não ao acaso.

\section{Discussão}

Através dos resultados obtidos pode-se constatar que a presente pesquisa atendeu as suas finalidades, apresentando escores significativos na melhora do déficit de atenção das crianças com TDAH, o que houve devido à eficácia da intervenção da estimulação cortical bem executada.

Quando analisados os resultados da segunda avaliação acredita-se que ao utilizar a frequência de $10 \mathrm{HZ}$ que, segundo Cardos09 está relacionada com a atenção e a aprendizagem, criou-se um cenário cortical propício a este tipo de evento, o que se pôde comprovar através da melhora no tempo de resposta a um estímulo.

Os resultados obtidos acerca da atenção concentrada, após a intervenção desenvolvida neste estudo corroboram com os resultados de pesquisadores como, Macedo et al. ${ }^{12}$ que também se valeram de estímulos auditivos simultâneos em seus trabalhos e assim como neste estudo, no que diz respeito a esta variável, também obtiveram escores significativos em sua pesquisa.

Em pesquisa realizada com crianças com TDAH, Schmiedeler e Schneider ${ }^{8}$, identificaram em seu estudo que os principais sintomas de desatenção estão negativamente associados às habilidades cognitivas na fase pré-escolar, onde os mesmos citam que assim que diagnosticados um problema nessa fase deve-se obter uma intervenção que seja eficaz para a melhora desse problema, fato esse proposto pela presente pesquisa que através da intervenção da estimulação cortical obteve melhoras significativas no déficit de atenção dos avaliados.

Em outro estudo que vem a colaborar com o presente estudo Bushe e Savill ${ }^{13}$ ao analisar índices de mortalidade em crianças com TDAH identificou a importância a qual se deve ter em realizar intervenções educacionais e mentais para a melhora da atenção e aspectos cognitivos desses indivíduos, onde quando estimulados cognitivamente podem apresentar melhoras de atenção, prevenindo futuros distúrbios psiquiátricos que influenciaram no seu processo de formação.

É propriamente neste mecanismo de maximização dos aspectos cognitivos aliados ao processo de atenção que atua a estimulação auditiva, permitindo condicionar a atividade cortical para melhorarmos um determinado desempenho, abrindo com isto as portas para que o córtex receba e interprete os diversos estímulos, selecionando as respostas mais adequadas para cada um deles e assim proporcionando benefícios na melhor da integração do sistema perceptivo motor $^{14}$. 
Já em intervenção diferentemente do presente estudo com atletas amadores, pode-se apontar o treinamento mental (bioperacional), via estimulação auditiva, como um promissor diferencial na performance e na aprendizagem dos estudados, diretamente ou por meios auxiliares, como na melhoria da atenção e da concentração, o que vem contribuir na melhora da integração do sistema perceptivo motor e comprovar a eficácia do método empregado na presente pesquisa ${ }^{15}$. Eficácia essa comprovada no cenário esportivo com atletas de futebol também, o que segundo Ribeiro Junior et al. ${ }^{16}$ afirma em seu estudo a importância da estimulação cortical na melhora do tempo de reação, na capacidade de raciocínio e na performance hábil motriz dos seus atletas identificando a relevância desse método na melhora da integração do sistema perceptivo motor.

Em estudo através da avaliação da informática educacional sendo aplicada simultaneamente com a estimulação cortical em crianças Beresford et al. ${ }^{17}$, promoveram uma melhora na adaptação dos mecanismos neurais do córtex responsável pela conexão intra-hemisférica, com isso melhorando a capacidade de atenção e concentração dos seus avaliados, o que vem a comprovar a eficácia desse método empregado em estudos dessa natureza.

\section{Conclusão}

Diante dos resultados obtidos neste estudo e por outros que também conduzem a mesma direção, pode-se concluir que a estimulação cortical, por via auditiva, realizada por meio de batidas binaural, mostrou-se valiosa na melhora dos níveis de atenção de crianças com TDAH.

Ao se minimizar a dificuldade de concentração dessas pessoas, busca-se com isto favorecer ao seu processo de aprendizagem, pois a estimulação cortical promoveu uma reorganização operacional dos mecanismos neurais responsáveis como: atenção, percepção, seleção e programação de respostas. Portanto, contribuiu para que os mesmos alcancem também outros níveis de desenvolvimento biopsicossociais.

Recomenda-se que sejam feita ademais pesquisas avaliando e maximizando o estudo de campo com a estimulação cortical e sua eficácia na melhora educacional e seus fatores como atenção em diversos públicos inclusive em crianças com TDAH onde são escassos estudos dessa natureza.

\section{Referências bibliográficas}

1. Rotta NT, Ohlweiler L, Riesgo RS. Transtornos da aprendizagem: abordagem neurobiológica e multidisciplinar. Porto Alegre: Artmed, 2006.

2. Posner MI, Rothbart MK. Influencing brain networks: implications for education. Trends in Cognitive Scienc. 2005;9(3):99103.

3. Arenal TA, Conde-Guzón PA. Formación de Subtipos de Niños con Problemas Escolares de Aprendizaje a Partir de la Evaluación Neuropsicológica, Capacidades Cognitivas y Comportamiento. Clínica y Salud. 2009;20(1).

4. Posner M, Fan J. Attention as an organ system. Sackler institute, weill medical college of Cornell university, 2006.

5. Massetti, G. M., Lahey, B. B., Pelham, W. E., Loney, J., Ehrhardt, A., Lee, S. S., \& Kipp, H. Academic achievement over 8 years among children who met modified criteria for attention-deficit/hyperac $\urcorner$ tivity disorder at 4-6 years of age. Journal of Abnormal Child Psychology, 2008;36:399-410.

6. Lubke GH, Hudziak JJ, Derks EM, Van Bijsterveldt T, Doomsma DI. Maternal ratings of attention problems in ADHD: Evidence for the existence of a continuum. Journal of American Academy of Child and Adolescent Psychiatry, 2009;48(11):1085-1093.

7. Arduini RG, Capellini SA, Ciasca SM. Comparative study of the neuropsychological and neuroimaging evaluations in children with dyslexia. Arquiv Neuropsiquiat. 2006;64(2):369-375.

8. Schmiedeler S, Schneider W. Attention-deficit hyperactivity disorder (ADHD) in the early years: Diagnostic issues and educational relevance. Clin Child Psycho and Psych. 2013;0(0):1-16.

9. Cardoso FB. A utilização do programa de potencialização cerebral para a melhoria no lançamento da bola de boliche para atletas da seleção juvenil colombiana B. Dissertação de Mestrado. Universidade Castelo Branco. Rio de Janeiro, 2007. 
10. Cardoso FB, Machado SEC, Silva VF. Estimulação Cerebral e Aprendizagem Motora: Efeitos no Aprendizado do Jogo de Boliche. Apresentado no III Congresso Científico Latino-Americano da FIEP, Foz do Iguaçu/PR, Brasil, 2006.

11. Thomas RJ, Nelson JK, Silverman SJ. Métodos de pesquisa em atividade física. Tradução Sales, D.R. Dornelles, M. S. 5. ed. Porto Alegre: Artmed, 2007.

12. Macedo LS et. al. O valor da estimulação cortical voltado para o déficit de atenção de alunos com síndrome de Down. Ciênc e cogn 2010;15(3):13-22.

13. Bushe CJ, Savill N. Suicide related events and attention deficit hyperactivity disorder treatments in children and adolescents: a meta-analysis of atomoxetine and methylphenidate comparator clinical trials. Child and Adolesc Psych and Ment Healt 2013;7(19):1-7.

14. Calomeni MR, Neto NTA, Leal KOF, Silva VF. Estimulação fótica e auditiva: Efeitos em criança hiperativa. Perspect onl 2008;5(2):34-42.

15. Neto NTA, Morales AP, Calomeni MR, Viana MAS, Silva VF. Estimulação cortical: efeitos agudos sobre variáveis bioperacionais em jogadores armadores de basquetebol. Rev dig, efdeportes.com, Buenos Aires 2010;15(150).

16. Ribeiro Júnior SMS, Azevedo MMA, Jorge FS, Morales AP, Silva VF. Efeito da estimulação cortical na variável tempo de reação e performance hábil-motriz-cognitiva em jovens atletas de futebol. Motr São Paulo, 2010;16(4):869-879.

17. Beresford H, Rosa AS, Rosa TS, Cardoso FB. O valor da informática educacional para a melhora do desenvolvimento lógico-matemático de crianças. Meta: Aval Rio de Janeiro 2010;2(4):60-72.

\section{Alisson Padilha de Lima}

Endereço para correspondência - Rua Silvio Romero, n.230, apto 108. Bairro Leonardo Ilha, CEP 99051-150, Passo Fundo, RS, Brasil.

E-mail: professor.alissonpadilha@gmail.com

Currículo Lattes: http://lattes.cnpq.br/8408892033315305

Fabricio B. Cardoso - fabriciobrunocardoso@gmail.com

Iris Lima e Silva - irislimauff@yahoo.com

Lucianne Fragel Madeira - lufragel@gmail.com

Alfred Sholl Franco - alfredsholl@gmail.com

Recebido em 08 de julho de 2013.

Publicado em 27 de feveiro de 2014. 